\title{
Immune Response to Recombinant Purified Hepatitis B Surface Antigen Vaccine among Health Care Workers in Jos, North Central Nigeria
}

\author{
Abba OJ ${ }^{1 *}$, Anejo-Okopi JA ${ }^{1}$, Akindgh $\mathrm{TM}^{1}$, Sambo $\mathrm{N}^{1}$, Pwol R ${ }^{1}$, Fadairo-Isah \\ $\mathrm{Y}^{1}$, Agbaji $\mathrm{OO}^{1,2}$, Oguche $\mathrm{S}^{1,3}$, Idoko $\mathrm{JA}^{4}$ \\ ${ }^{1}$ AIDS Prevention Initiative in Nigeria, Jos University Teaching Hospital, Jos Nigeria \\ ${ }^{2}$ Department of Medicine, University of Jos, Jos, Nigeria \\ ${ }^{3}$ Department of Paediatrics, University of Jos, Jos, Nigeria \\ ${ }^{4}$ National Agency for Control of AIDS, Abuja, Nigeria
}

\begin{abstract}
:
Background: Seroconversion rates reported after Hepatitis $B$ virus (HBV) vaccination globally ranges from 85-90\%. Health care workers (HCW) in health institutions are at risk of acquiring hepatitis B infection. Therefore, this study determined through qualitative evaluation of immune responses to recombinant purified hepatitis $B$ surface antigen vaccine among health care workers in Jos, North central Nigeria.

Methods: Health care workers who had received recombinant purified HBsAg vaccine at the Jos University Teaching Hospital (JUTH), AIDS Prevention in Nigeria (APIN) supported HIV were evaluated for immune response through qualitative method 6 weeks post-vaccination. Descriptive observational study design was used in this survey from April 2012 to June 2013

Results: Study subjects (HCW) were 115. Females were 68 (59.1\%), males were 47(40.9\%). Study participants were between the ages of 25-34 years (51.3\%) regardless of gender. Out of the 115 participants examined 99(86.1\%) had established HBsAb while 16(13.9\%) remained seronegative after six weeks of post vaccination. The mean age of responders was $36.0 \pm 6.9$ years.

Conclusion: Our study showed that majority of the HCW at APIN JUTH, who received the purified recombinant HBsAg vaccine had immunologic response. This response is however varied according to age groups. The study participants who are aged less than 44 years were more likely to respond satisfactorily. These underscore the importance of checking immune response to this vaccine among $H C W$, to ensure they are protected against nosocomial acquisition of $H B V$. Health care workers who fail to respond to vaccination after standard dosing should be given a fourth dose or the entire course of vaccination repeated.
\end{abstract}

Keywords: Hepatitis B virus, Vaccine, anti-HBs, Qualitative

\section{Introduction}

Hepatitis B virus (HBV) infection and its resulting conditions, including chronic liver disease, cirrhosis and hepatocellular carcinoma are major global health problems. It is estimated that 2 billion of the world's population has been infected with the hepatitis B virus and about 350 million people are reported to be chronically infected with the virus worldwide and constitute the primary reservoir of infection ${ }^{1}$.

Prevention of health care workers (HCWs) from the acquisition of hepatitis B virus (HBV) infection; especially those that are in frequent contact with blood are a major public health concern. Health care workers in health institutions especially those that cater for infectious diseases are at high risk of acquiring the HBV infection and possibly becoming chronically infected, the risk of acquisition of HBV infection after a single exposure of HBV infected blood or body fluid ranges from $6 \%-30 \%{ }^{2,3,4}$ In addition to health education directed toward decreasing high risk behavior, vaccination is the best tool in protecting HCWs. Since the availability of HBV vaccine from 1982, the decline in the incidence of HBV infection and associated morbidity and mortality has been reported $5,6,7,8$. Therefore, in $1997 \mathrm{CDC}$ recommended that all HCWs should be vaccinated against $\mathrm{HBV}^{9}$. Despite the recommendation and excellent protection profile among $\mathrm{HCWs}$ vaccinated personnel, compliance to this recommendation remained poor in various health care settings ${ }^{10,11}$. Factors associated with decreased immune response to HBV vaccine include: increasing age, smoking, obesity, gender and genetic factors ${ }^{14-16}$. Studies on HCWs published from various parts of the world have reported 12$21 \%$ non responders to HBV vaccine. Despite HBV infection being a major health care issue in both community and nosocomial settings in Nigeria, information on the qualitative immune response in HCWs is scarce. Therefore, we determined the immune responses to recombinant purified Hepatitis B surface antigen vaccine among HCWs in Jos, North central Nigeria using qualitative technique. 


\section{Methods}

Serum samples from HCWs were screened for HBsAg those that were negative, who consented to be part of the study had HBV vaccine $1 \mathrm{ml}(20 \mathrm{mcg})$ administered intramuscularly in the deltoid muscle at months 0 , 1, and 6 respectively. Eight weeks later, five milliliters of their venous blood was collected into container with EDTA. The plasma separated immediately by placing the specimen EDTA bottle on a standing position in a rack. Testing for antibodies was performed using the Rapid HBV COMBO test kit Acumen Diagnostic Inc. U.S.A according to manufacturer's instructions. Data was analyzed using SPSS version 17.

\section{Results}

A total of $115 \mathrm{HCWs}$ were enrolled for this study. Females were 68(59.1\%) and males were $47(40.9 \%)$. The overall response rate of health care workers at the APIN center to the Hepatitis B vaccine after 3 doses and six weeks post vaccination was 99 (86.1\%) (Table 1) and (Fig 1). After six weeks post vaccination, $13.9 \%$ of the HCWs did not develop HBsAb and were tagged non-respondents. (Fig1). Majority of the study participants were between the ages of 25-44 years with mean age of study participants $37.4 \pm 7.5$ years. The age group of workers between 25-34years and 35-44 years showed the highest response (84.3\%) to the vaccine establishing $\mathrm{HBsAb}$ after 6 weeks post vaccination. The mean age of male non responders was higher than that for females, $43 \pm 15.7$ years and $34.7 \pm 2.75$ years respectively and also showed a significantly reduced response to the vaccine than females $(\mathrm{P}<0.05,95 \% \mathrm{CI}=4.9)$

Table 1: Demographic characteristics of study subjects

\begin{tabular}{lccl}
\hline & n after $\mathbf{3}$ doses $\mathbf{( \% )}$ & n respondents (\%) & Non- respondents (\%) \\
\hline & & & \\
Total & 115 & $99(86.1)$ & $16(13.9)$ \\
Males & $47(40.9)$ & $39(39.2)$ & $8(6.9)$ \\
Females & $68(59.1)$ & $60(58.8)$ & $8(50.0)$ \\
25-34 years & $48(100)$ & $42(87.5)$ & $6(12.5)$ \\
$\mathbf{3 5 - 4 4}$ years & $50(100)$ & $41(82.0)$ & $9(18.0)$ \\
$\mathbf{4 5 - 4 9}$ years & $10(100)$ & $10(100)$ & 0 \\
$\mathbf{2 5 0}$ years & $7(100)$ & $6(85.7)$ & $1(14.3)$ \\
\hline
\end{tabular}

Table 2: Immune Response according Age and sex distribution of study subjects

\begin{tabular}{|c|c|c|c|c|c|}
\hline \multicolumn{6}{|c|}{ Responders (n \%) } \\
\hline & & $25-34$ yrs & $35-44 \mathrm{yrs}$ & $45-49 y r s$ & $\geq 50 \mathrm{yrs}$ \\
\hline Female & $\mathrm{n}=60$ & $24(24.2)$ & $27(27.3)$ & $6(6.1)$ & $3(3.0)$ \\
\hline Male & $n=39$ & $18(18.2)$ & $14(14.1)$ & $4(4.0)$ & $3(3.0)$ \\
\hline \multicolumn{6}{|c|}{ Non- Responders (n \%) } \\
\hline & & $25-34$ yrs & $35-44 y r s$ & $45-49 y r s$ & $\geq 50 \mathrm{yrs}$ \\
\hline Female & $\mathrm{n}=8$ & $4(25.0)$ & $3(18.7)$ & 0 & $1(6.3)$ \\
\hline Male & $\mathrm{n}=8$ & $2(12.5)$ & $6(37.5)$ & 0 & 0 \\
\hline
\end{tabular}

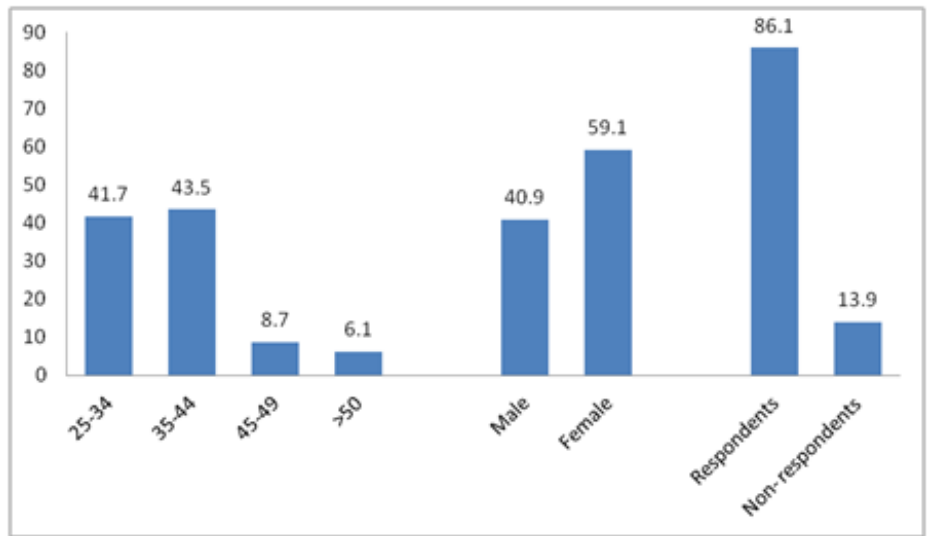

Figure 1: Descriptive distribution of Age, sex and Response

\section{Discussion}

In this study, 99 (86.1\%) of study participants established HBsAb after six weeks post vaccination while $13.9 \%$ remained non-respondents failing to establish the HbsAb after recommended routine HBV vaccination. From a public health standpoint, these results indicate that a follow up test post vaccination is an 
important strategy to employ in the safety of health care workers. These findings are similar to those reported by Zeeshan et al. (2007) who observed $86.2 \%$ responders and $13.2 \%$ non-responders among HCWs in Pakistan. ${ }^{21}$ Roome et al. ${ }^{1}$. (1993), Averhoff et al. ${ }^{22}$ (1998) and Saberiflroozi et al. ${ }^{23}$ (2006) have reported $11.9 \%, 12 \%$ and $12.7 \%$ non-respondents respectively in the USA and Iran

In this study, age and gender were the two variables considered. Among respondents $84.3 \%$ ( 86) were between the ages of 25-44years (Table 2). A study carried out in Sweden on response rates after intradermal vaccination in HCWs observed a higher response in subjects below the age of 40 and a significantly lower response in subjects older than 40years ${ }^{27}$. Similarly, the frequency of non- respondents in our study were also from study participants between the ages of 25-44 years (Table 2). While it has been shown that increase in nonrespondent age increases with age, this may be explained by other risk factors such as smoking, body mass index and alcohol intake which were not evaluated by our study.

Male non- respondents showed a significantly reduced response to the vaccine than female nonrespondents $(\mathrm{P}<0.035)$. Smoking and other genetic factors which could contribute to poor response in men have not been explored in this study. In Pakistan, a study among HCWs showed that the frequency of male nonresponders was higher than female non-responders $18 \%$ and $9 \%$ respectively $(p=0.001)$.

The frequency of occurrence for male non-responders in this study was $6.9 \%$ and $6.9 \%$ for females. The results of this study suggest that both male and female health care workers are at equal risk of exposure to HBV. This result differs from the findings reported by Wood et al 1993 and Zeeshan et al.(2007) who observed a response rate of $18 \%$ and $9 \%$ respectively of male and female $(P=0.006$ and 0.001 respectively). A recent study showed lower response in healthy homosexual men compare to healthy workers ${ }^{17}$ also an association with different HLA-DR alleles has been found in different studies, those with HLA phenotype of B44, DRB1*0701, DQB1 *0201 were nearly four times likely to be non-respondents compared to respondents. It is possible that the response may be specific to HBV surface antigen recognition. The ability to produce antibody in response to a specific protein is controlled by dominant autosomal class II genes of the major histocompatability complex (MHC) ${ }^{24}$ Smoking and certain genetic factors have been reported as probable reasons of decreased immune response in male. ${ }^{18}$ Uppermost rate of immune response was observed among those with age group of 25 44years and started decline with increasing in age $(p=0.0001)$. Among HCWs $>50$ years of age, only $6.9 \%$ developed response to HBV vaccine, though the sample size in this group was small, the findings are more or less in agreement with Roome et al's (1993) observation of inadequate levels of antibodies in relation to increasing age, from $2.8 \%$ among those younger than 30years to $42.1 \%$ among those older than 60 years $(p<0.0001)$ of age ${ }^{16}$. This finding has great clinical significance as non-responders remain susceptible to HBV infections. Therefore, from an infection control perspective, the post vaccination HbsAb establishment should be determined for all HCWs. In Nigeria, there is limited data regarding immune response after HBV vaccination in HCWs, this study therefore adds to the growing body of reports on immune responses to HBV vaccine in $\mathrm{HCWs}$ from different parts of the world.

A limitation of this study was the absence of a quantitative determination of anti-HBs titres. However, in a resource limited setting our results provide a baseline for future epidemiological studies in this area. Also we did not evaluate the association of decreased immune response with risk factors other than age and gender. Struve et al $(1992)^{25}$ and Winter et al $(1994)^{26}$ have suggested smoking as a risk factor in poor response to HBV vaccine. Obesity defined as a BMI $>30$ has been suggested to increase the risk of vaccine failure as observed in the very obese with BMI over thirty five ${ }^{16}$. Immunosuppression has also been reported by some authors to result in reduced response rates to vaccination. The response to vaccine in HIV infected patients has been shown to be related to low $\mathrm{CD}^{+}$counts and high HIV-RNA levels. ${ }^{28}$, Other factors that have been mentioned are nutritional status, site of administration of vaccine, and immunogenetic make up also contributed to reduced immune response.

\section{Conclusion}

The use of qualitative method to determine the immunological response among HCWs can be adopted among health institution bearing in mind the economic burden when using the quantitative technique. In this study we were able to establish that HCWs with $\mathrm{HBcAb}$ responded well to the establishment of HbsAb using the qualitative technique. There is a need to strictly implement the policy of hepatitis B immunization in every health care location as recommended by CDC. It is also imperative to check the post vaccination status of all HCWs after 6-8 weeks of vaccination as it not ensure safety of employees but also reduces rate of transmission hence functioning as a cost effective exercise at individual as well as national level. This is the first qualitative study that provides the local epidemiological information assessing the immune response to HBV vaccine among HCWs in Nigeria. 


\section{Recommendation}

Post vaccination testing is recommended for high risk person, including health-care and public safety workers; chronic hemodialysis patients, HIV-infected persons, and other immunocompromised persons, and sex or needle-sharing partners of HBsAg-positive persons. Though, in majority of health institutions in Nigeria this practice is usually not followed ${ }^{20}$. The importance of pre-exposure vaccination cannot be over emphasize because of the cost effectiveness that the post exposure which cost more and the extra cost burden could either be on the HCWs or the institution especially in the developing world.

\section{Conflict of Interest}

None

\section{Acknowledgements}

We are grateful to the JUTH APIN management for their support and also to the entire staff of the centre for their cooperation and technical contribution in this study.

\section{References}

[1]. JH Kao, DS Chen: Overview of Hepatitis B and C virus. In infectious cause of cancer: target for intervention. Edited by Guerdon TJJ. Totowa N.J: Humana press; 2000:313-30.

[2]. E Rosea, B Rudensky, E Pez: Ten years follow up study of hepatitis B virus infection and vaccination status in hospital employee. J Hosp Infec 1999, 41:245-50

[3]. M Tallat, 1 AKandee, W El-Shoubory, C Bodenschatz, I K hairy, S Oun,.: Occupational exposures to needle stick injuries and hepatitis B vaccination coverage among health care workers in Egypt. Am J Infect Control 2003, 31 (8):469-74.

[4]. A Kane, J Lioyd, M Zaffran, L Simonsen, M Kane: Transmission of hepatitis B, hepatitis C and human immunodeficiency viruses through unsafe injections in the developing world: model-based regional estimates. Bull World Health organ1999, 77:801-7.

[5]. YH Ni, MH Chang, LM Huang, HL Chen, HY Hsu, TY Chiu, et al: Hepatitis B virus infection in children and adolescent in a hyperendemic area: 15 years after mass hepatitis B vaccination. Ann Med 2001, 135(9):796-800.

[6]. RN Gunson, GL Sorald, FM Roggrndor, H Nicholas: Hepatitis B virus and hepatitis C virus infection in health care workers: guideline for prevention of transmission of HBV and HCV from HCW to patient. J Clin Virol 2003, 27:213-30.

[7]. BP Lanpher, CC Jr Linnemann, CG Cannon, MM Deroub: Decline of clinical hepatitis B in workers at a general hospital: Relations to increase vaccine induce immunity. CID1993, 16(16):10-14.

[8]. FJ Mahoney, K Stewart, H Hu, P Coleman, MJ: Alter Progress toward the elimination of hepatitis B virus transmission among health care workers in the United States. Arch Intern Med 157(22):2601-2605.

[9]. "Immunization of health care workers" Recommendation of advisory committee on immunization practice (ACIP) and the Hospital Infection Control Practice Advisory committee (HICPAC). MMWR, Recommendation and report 26: 12/26/97.

[10]. SK Shrestha, 1 M.DBhattara: Study of Hepatitis B among different categories of health care workers. J Coll Physicians Surg Pak 2006, 16(2): 108-11.

[11]. E Dannetun, A Teqneu, A Torner, J Giesecke: Coverage of hepatitis B vaccination in Swedish health care workers. J Hosp Infect 2006, 63(2): 201-4.

[12]. AD Jack, AJ Hall, N Maine, M Meudy, HC Whittle: What level of hepatitis B antibody is protective? J Infect Dis 1999, 179:489492.

[13]. PAG Maupas, DF Simon: Immunization against hepatitis B. Lancet 1988, 2:875-876.

[14]. EB Hollinger, CN Kim HU, Lee: Factor influencing the immune response to hepatitis B vaccine, booster dose guideline and vaccine protocol recommendation. Am J Med 1989, 16:365-403

[15]. RC Wood, KL MacDonald, KE White, GW Hedbert, M Harrison: Risk factors for lack of detectable antibody following hepatitis B vaccination of Minnesota Health care workers.JAMA 1993, 270:2935-9

[16]. AJ Roome, SJ Walsh, ML Catter JL, Haddle: Hepatitis B vaccine responsiveness in Connecticut public safety personnel. JAMA 1993, 270: 293 1-4.

[17]. JN Zuckerman, AJ Zuckerman. Current topics in Hepatitis B. Journal of Infection 2000;41: 130-36

[18]. YH Yen, C-H Chen, J-H Wang, C-M Lee, C-S Changchien, S-N Lu: Study of hepatitis B vaccine non-responsiveness among health care workers from endemic area (Taiwan). Liver International 2005, 25:1162-1168.

[19]. NS Ali, K Jamal, R Qureshi: Hepatitis B vaccination status and identification of risk factors for hepatitis B in health care workers. J Coll Physicians Surg Pak 2005, 15 (5): 257-260.

[20]. SA Mujeeb, Y Khatri, R Khanani: Frequency of Parenteral exposure and seroprevalence of HBV, HBC and HIV among operation room personnel. J Hosp infect 1998, 38:133-7

[21]. M Zeeshan, K Jabeen, ANA Ali, WA Ali, SZ Farooqui, V Mehraj, A Zafar: Evaluation of immune response to Hepatitis B vaccine in Health care workers at a tertiary hospital in Pakistan: an observational prospective study. BMC Inf. Dis 2007, 7:120

[22]. F Averhoff, F Mohoney, P Coleman, G Schatz, E Hurwitz, H Margolis: Immunogenecity of Hepatitis B vaccines: Implications for persons at occupational risk of Hepatitis B virus infection. Am J Prev Med 1998, 15(1):73-7

[23]. M Saberifiroozi, S Gholamzadeh, AR Serati: The long-term immunity among health workers vaccinated against hepatitis B virus in a large referral hospital in southern Iran. Arch Iran Med 2006, 9(3):204-7

[24]. MS Kruskall, CA Alper, Z Awdeh, E Yunis, D Marcus-Bagley:The immune response to hepatitis B vaccine in humans. Inheritance patterns in families. J Exp. Med 1992, (175): 495-502

[25]. J Struve, B Aronsson, B Frenning, F Granath, Von M Sydow and O Weidow: Intramuscular versus intradermal administration of a recombinant hepatitis B vaccine: a comparison of response rates and analysis of factors influencing the antibody response. Scand. $\mathrm{J}$ Infect Dis. 1992, 24(4):423-9

[26]. AP Winter, EA Follett, J McIntyre, J Stewart, and IS Symington: Influence of smoking on immunological responses to hepatitis B vaccine. Vaccine 1994, 12(9):771-2

[27]. K Cardell, A Fryden and B Normann: Intradermal hepatitis B vaccination in health care workers. Response rate and experiences from vaccinations in clinical practice. Scand J Infect Dis. 1999; 31:197-200

[28]. EM RK Tedaldi, Baker, AC Moorman, et al.: Hepatitis A and B vaccination practices for ambulatory patients infected with HIV. Clin Infect Dis. 2004;38(10):1478-84 